\title{
Basic science: (April 2009)
}

1. Alli E, Sharma VB, Sunderesakumar P, Ford JM. Defective repair of oxidative DNA damage in triple-negative breast cancer confers sensitivity to inhibition of poly(ADP-ribose) polymerase. Cancer Res 2009; 69: 3589-3596.

2. Andre F, Michiels S, Dessen P, Scott V, Suciu V, Uzan C, Lazar V, Lacroix L, Vassal G, Spielmann $M$, Vielh $P$, Delaloge $S$. Exonic expression profiling of breast cancer and benign lesions: a retrospective analysis. Lancet Oncol 2009; 10: 381-390.

3. Azuma K, Urano T, Horie-Inoue K, Hayashi S, Sakai R, Ouchi $Y$, Inoue S. Association of estrogen receptor $\alpha$ and histone deacetylase 6 causes rapid deacetylation of tubulin in breast cancer cells. Cancer Res 2009; 69: 2935-2940.

4. Barclay JL, Anderson ST, Waters MJ, Curlewis JD. SOCS3 as a tumor suppressor in breast cancer cells, and its regulation by PRL. Int $J$ Cancer 2009; 124: 1756-1766.

5. Cardamone MD, Bardella C, Gutierrez A, Croce LD, Rosenfeld MG, Di Renzo MF, De Bortoli M. $\mathrm{ER} \alpha$ as ligand-independent activator of $\mathrm{CDH}-1$ regulates determination and maintenance of epithelial morphology in breast cancer cells. Proc Natl Acad Sci U S A 2009; 106: 7420-7425.

6. Cheng CJ, Lin YC, Tsai MT, Chen CS, Hsieh MC, Chen CL, Yang RB. SCUBE2 suppresses breast tumor cell proliferation and confers a favorable prognosis in invasive breast cancer. Cancer Res 2009; 69: 3634-3641.

7. De Amicis F, Zupo S, Panno ML, Malivindi R, Giordano F, Barone I, Mauro L, Fuqua SAW, Ando S. Progesterone receptor $B$ recruits a repressor complex to a half-PRE site of the estrogen receptor $\alpha$ gene promoter. $\mathrm{Mol}$ Endocrinol 2009; 23: 454-465.

8. Delk NA, Hunt KK, Keyomarsi K. Altered subcellular localization of tumor-specific cyclin $\mathrm{E}$ isoforms affects cyclin-dependent kinase 2 complex formation and proteasomal regulation. Cancer Res 2009; 69: 2817-2825.

First published online 14/07/09

BCO/853/2009/JW
9. Gonzalez-Angulo AM, Stemke-Hale K, Palla SL, Carey M, Agarwal R, Meric-Berstam F, Traina TA, Hudis C, Hortobagyi GN, Gerald WL, Mills GB, Hennessy BT. Androgen receptor levels and association with PIK3CA mutations and prognosis in breast cancer. Clin Cancer Res 2009; 15: 2472-2478.

10. Habermann JK, Doering J, Hautaniemi S, Roblick UJ, Bundgen NK, Nicorici D, Kronenwett $\mathrm{U}$, Rathnagiriswaran S, Mettu RKR, Ma Y, Kruger S, Bruch HP, Auer G, Guo NL, Ried T. The gene expression signature of genomic instability in breast cancer is an independent predictor of clinical outcome. Int $J$ Cancer 2009; 124: 1552-1564.

11. Kataoka M, Antoniou A, Warren R, Leyland J, Brown J, Audley T, Easton D. Genetic models for the familial aggregation of mammographic breast density. Cancer Epidemiol Biomarkers Prev 2009; 18: 1277-1284.

12. Kazi A, Carie A, Blaskovich MA, Bucher C, Thai V, Moulder S, Peng H, Carrico D, Pusateri E, Pledger WJ, Berndt N, Hamilton A, Sebti SM. Blockade of protein geranylgeranylation inhibits Cdk2-dependent p27(Kip1) phosphorylation on Thr187 and accumulates p27(Kip1) in the nucleus: implications for breast cancer therapy. Mol Cell Biol 2009; 29: 2254-2263.

13. Kwek SS, Roy R, Zhou H, Climent J, MartinezCliment JA, Fridlyand J, Albertson DG. Coamplified genes at $8 p 12$ and $11 q 13$ in breast tumors cooperate with two major pathways in oncogenesis. Oncogene 2009; 28: 1892-1903.

14. Mercier I, Casimiro MC, Zhou J, Wang C, Plymire C, Bryant KG, Daumer KM, Sotgia F, Bonuccelli G, Witkiewicz AK, Lin J, Tran TH, Milliman J, Frank PG, Jasmin JF, Rui H, Pestell RG, Lisanti MP. Genetic ablation of caveolin-1 drives estrogen-hypersensitivity and the development of DCIS-like mammary lesions. Am J Pathol 2009; 174: 1172-1190.

15. Millar EKA, Dean JL, McNeil CM, O'Toole SA, Henshall SM, Tran T, Lin J, Quong A, Comstock CES, Witkiewicz A, Musgrove EA, Rui $\mathrm{H}$, LeMarchand L, Setiawan VW, Haiman CA, 
Knudsen KE, Sutherland RL, Knudsen ES. Cyclin D1b protein expression in breast cancer is independent of cyclin D1a and associated with poor disease outcome. Oncogene 2009; 28: $1812-1820$.

16. Natrajan $R$, Lambros $M B$, Rodriguez-Pinilla SM, Moreno-Bueno G, Tan DSP, Marchio C, Vatcheva R, Rayter S, Mahler-Araujo B, Fulford LG, Hungermann D, Mackay A, Grigoriadis A, Fenwick K, Tamber N, Hardisson D, Tutt A, Palacios J, Lord CJ, Buerger H, Ashworth A, Reis JS. Tiling path genomic profiling of grade 3 invasive ductal breast cancers. Clin Cancer Res 2009; 15: 2711-2722.

17. Neal CL, Yao J, Yang WT, Zhou XY, Nguyen NT, Lu J, Danes CG, Guo H, Lan KH, Ensor J, Hittelman W, Hung MC, Yu DH. 14-3-3 $\zeta$ Overexpression defines high risk for breast cancer recurrence and promotes cancer cell survival. Cancer Res 2009; 69: 3425-3432.

18. Nikkila J, Coleman KA, Morrissey D, Pylkas K, Erkko $H$, Messick TE, Karppinen SM, Amelina A, Winqvist R, Greenberg RA. Familial breast cancer screening reveals an alteration in the RAP80 UIM domain that impairs DNA damage response function. Oncogene 2009; 28: 1843-1852.

19. Ohashi E, Kogai T, Kagechika H, Brent GA. Activation of the PI3 kinase pathway by retinoic acid mediates sodium/iodide symporter induction and iodide transport in MCF-7 breast cancer cells. Cancer Res 2009; 69: 3443-3450.

20. Pitroda SP, Khodarev NN, Beckett MA, Kufe DW, Weichselbaum RR. MUC1-induced alterations in a lipid metabolic gene network predict response of human breast cancers to tamoxifen treatment. Proc Natl Acad Sci U S A 2009; 106: $5837-5841$.

21. Plotnikov A, Varghese B, Tran TH, Litt CB, Rui $\mathrm{H}$, Fuchs SY. Impaired turnover of prolactin receptor contributes to transformation of human breast cells. Cancer Res 2009; 69: 3165-3172.

22. Rakha EA, Elsheikh SE, Aleskandarany MA, Habashi HO, Green AR, Powe DG, El-Sayed ME, Benhasouna A, Brunet JS, Akslen LA, Evans AJ, Blamey R, Reis JS, Foulkes WD, Ellis IO. Triple-negative breast cancer: distinguishing between basal and nonbasal subtypes. Clin Cancer Res 2009; 15: 2302-2310.
23. Santisteban M, Reiman JM, Asiedu NK, Behrens MD, Nassar A, Kalli KR, Haluska P, Ingle JN, Hartmann LC, Manjili MH, Radisky DC, Ferrone S, Knutson KL. Immune-induced epithelial to mesenchymal transition in vivo generates breast cancer stem cells. Cancer Res 2009; 69: 2887-2895.

24. Shackleton M, O'Reilly LA, Sutherland KD, Bath ML, Ellis S, Strasser A, Visvader JE, Lindeman GJ. Impaired lactation in mice expressing dominant-negative FADD in mammary epithelium. Dev Dyn 2009; 238: 1010-1016.

25. Shephard ND, Abo R, Rigas SH, Frank B, Lin WY, Brock IW, Shippen A, Balasubramanian SP, Reed MWR, Bartram CR, Meindl A, Schmutzler RK, Engel C, Burwinkel B, Cannon-Albright LA, Allen-Brady K, Camp NJ, Cox A. A breast cancer risk haplotype in the caspase-8 gene. Cancer Res 2009; 69: 2724-2728.

26. Wang B, Mi MT, Wang J, Wei N, Zhang QY, Zhu JD, Yang S, Guo BT, Xu J, Yang XH. Does the increase of endogenous steroid hormone levels also affect breast cancer risk in Chinese women? A case-control study in Chongqing, China. Int J Cancer 2009; 124: 1892-1899.

27. Wang MY, Chen PS, Prakash E, Hsu HC, Huang HY, Lin MT, Chang KJ, Kuo ML. Connective tissue growth factor confers drug resistance in breast cancer through concomitant up-regulation of Bcl-xL and clAP1. Cancer Res 2009; 69: 3482-3491.

28. Workman HC, Sweeney C, Carraway KL. The membrane mucin Muc4 inhibits apoptosis induced by multiple insults via ErbB2-dependent and ErbB2-independent mechanisms. Cancer Res 2009; 69: 2845-2852.

29. Wua M, Jung L, Cooper AB, Fleet $C$, Chen LH, Breault L, Clark K, Cai ZH, Vincent S, Bottega S, Shen Q, Richardson A, Bosenburg M, Naber SP, DePinho RA, Kuperwasserf C, Robinson $M O$. Dissecting genetic requirements of human breast tumorigenesis in a tissue transgenic model of human breast cancer in mice. Proc Natl Acad Sci U S A 2009; 106: 7022-7027.

Prepared by $R$ Sutherland Cancer Research Program Garvan Institute of Medical Research Darlinghurst, NSW, Australia 